\title{
Small-Strip Thin Gap Chambers for the Muon Spectrometer Upgrade of the ATLAS Experiment
}

\author{
Auriane Canesse ${ }^{a, *}$ on behalf of the ATLAS Muon Collaboration \\ ${ }^{a}$ McGill University, \\ 3600 Rue University, Montréal, Canada \\ E-mail: auriane.canesse@cern.ch
}

\begin{abstract}
The instantaneous luminosity of the Large Hadron Collider at CERN will be increased by about a factor of five with respect to the design value by undergoing an extensive upgrade program over the coming decade. The largest phase-1 upgrade project for the ATLAS Muon System is the replacement of the present first station in the forward regions with the New Small Wheels (NSW) during the long LHC-shutdown in 2019-2021. Along with Micromegas detectors, the NSW will be equipped with eight layers of small-strip thin gap chambers (sTGC) arranged in multilayers of two quadruplets, for a total active surface of more than $2500 \mathrm{~m}^{2}$. To retain the good precision tracking and trigger capabilities in the high background environment of the High-Luminosity LHC, each sTGC plane must achieve a spatial resolution better than $100 \mu \mathrm{m}$ to allow the Level-1 trigger track segments to be reconstructed with an angular resolution of approximately 1 mrad. The basic sTGC structure consists of a grid of gold-plated tungsten wires sandwiched between two resistive cathode planes at a small distance from the wire plane. The precision cathode plane has strips with a $3.2 \mathrm{~mm}$ pitch for precision readout and the cathode plane on the other side has pads for triggering. The sTGC design, performance, construction and integration status is discussed, along with results from tests of the chambers with nearly final electronics with beams, cosmic rays and high-intensity radiation sources.
\end{abstract}

The Eighth Annual Conference on Large Hadron Collider Physics-LHCP2020

25-30 May, 2020

online

\footnotetext{
${ }^{*}$ Speaker
} 


\section{Introduction}

The ATLAS [1] New Small Wheels (NSW) will replace the current first station of the ATLAS muon end-cap system [2]. They are designed to provide a $\sim 7$ fold increase in rejection rate for fake muon triggers and an improved muon momentum measurement at the High-Luminosity LHC (HLLHC). The NSW exploit two detector technologies: the Micromegas (MM) detectors [3] optimized for precision tracking and the small-strip Thin Gap Chambers (sTGC) [4] optimized for triggering. One wheel is made of 16 sectors and each sector is composed of two sTGC wedges and one MM double-wedge as shown in Figure 1. The sTGC wedges are made of three quadruplets modules, each composed of four sTGC layers.

Small-strip Thin Gap Chambers are multiwire ionization chambers operated in quasi-saturated mode. Each layer is made of segmented cathodes and anode wires (see Figure 2). One cathode is divided into large pads used for triggering: a 3 -out-of- 4 coincidence within a quadruplet (4 layers) is required. The other cathode is divided into strips with a $3.2 \mathrm{~mm}$ pitch for precision muon track reconstruction in the $\eta$ direction. The sTGC are operated at a voltage of $2.8 \mathrm{kV}$ with a 55:45 gas mixture of $\mathrm{CO}_{2}$ and n-pentane. Track segments are reconstructed from charge clusters of typically 3 to 5 strips. The cluster centroid position is obtained from a Gaussian fit of the strip pulse height distribution.
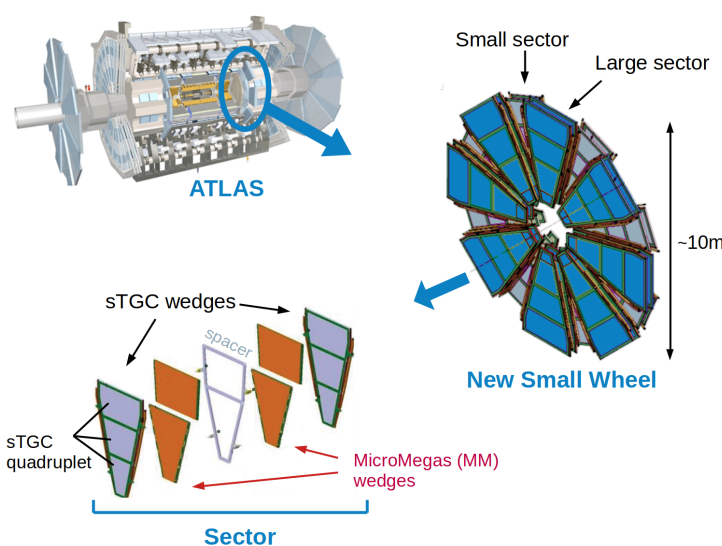

Figure 1: Schematic diagram showing the location of sTGC on the NSW and within the ATLAS detector.

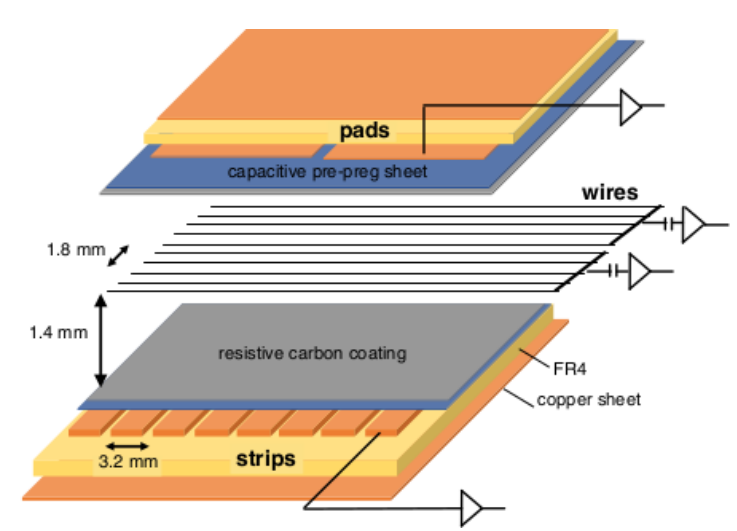

Figure 2: Schematic diagram of a sTGC [2].

\section{Spatial resolution measurements}

Cosmic rays study: The spatial resolution of a $40 \times 60 \mathrm{~cm}^{2}$ sTGC quadruplet prototype equipped with prototype front-end readout electronics was measured using cosmic rays. Scintillator detector planes were used to provide a trigger signal [5]. To measure the prototype's spatial resolution, track segments were reconstructed using hits from 3 and from all 4 layers. Inclusive and exclusive track fit residuals were obtained after correcting for inter-layer misalignment. The spatial resolution is obtained from the geometric mean of the inclusive and exclusive residual distributions widths. The 
spatial resolution can be parameterized as a function of the cosmic ray incidence angle $\theta_{y z}$ :

$$
\sigma_{s T G C}^{2}=\sigma_{0}^{2}+\sigma_{\theta y z}^{2} \tan \theta_{y z}^{2}
$$

where $\sigma_{0}$ is the intrinsic spatial resolution of muons perpendicular to the detector surface and $\sigma_{\theta y z}$ the contribution of charge production fluctuations along the axis perpendicular to the strips. The spatial resolution varies from approximately $100 \mu \mathrm{m}$ to $300 \mu \mathrm{m}$ depending on the muon angle as shown in Figure 3.

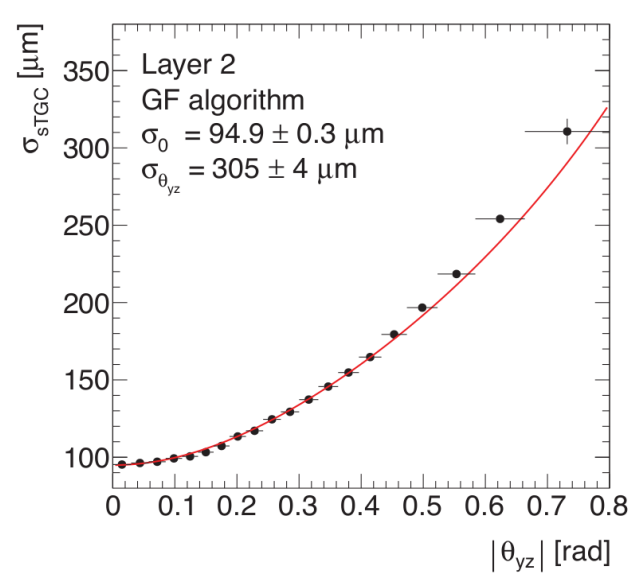

Figure 3: Spatial resolution of a sTGC prototype as a function of the cosmic muon incidence angle [5].

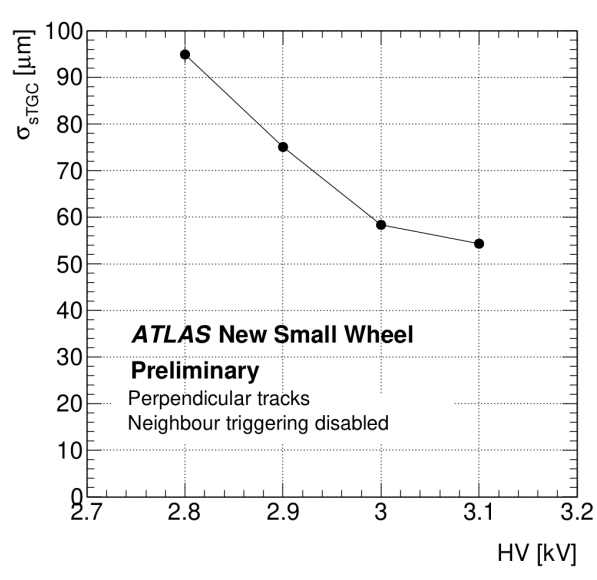

Figure 4: sTGC strip spatial resolution as a function of the applied high-voltage measured using a low-rate muon beam in the $\mathrm{H} 8$ beam-test area at CERN [6].

Test beam measurements: To measure the spatial resolution of sTGC production modules, a low-rate muon beam was used in the $\mathrm{H} 8$ beam-test area at CERN [6]. The sTGC strip spatial resolution for one layer as a function of the applied high-voltage was measured and is shown in Figure 4 . The measured resolution at the nominal voltage of $2.8 \mathrm{kV}$ is $\sim 95 \mu \mathrm{m}$ which is better than the minimum requirement of $100 \mu \mathrm{m}$.

The sTGC detectors have also been exposed to the muon beam within a high photon radiation background in the GIF++ facility at CERN [7]. The photons are produced through decays of a high intensity ${ }^{137} \mathrm{Cs}$ source placed $\sim 2 \mathrm{~m}$ away from the detector's surface. The photon rate can be remotely controlled with a set of movable shielding blocks placed near the source. The sTGC pad charge distribution measured as a function of photon rate is shown in Figure 5. A clear separation from the noise pedestal can be seen for all photon rates up to $\sim 10 \mathrm{kHz} / \mathrm{cm}^{2}$. The HL-LHC background rate for this sTGC module is expected to be in the range of $\sim 3 \mathrm{kHz} / \mathrm{cm}^{2}$ to $10 \mathrm{kHz} / \mathrm{cm}^{2}$. Note that in the NSW, the muon incidence angle will in general not be perpendicular to the sTGC and the resolution is expected to worsen (see Figure 3). However, the front-end electronics used for these test-beam measurements were demonstrator versions and small improvements are expected with the final version of the electronics. 


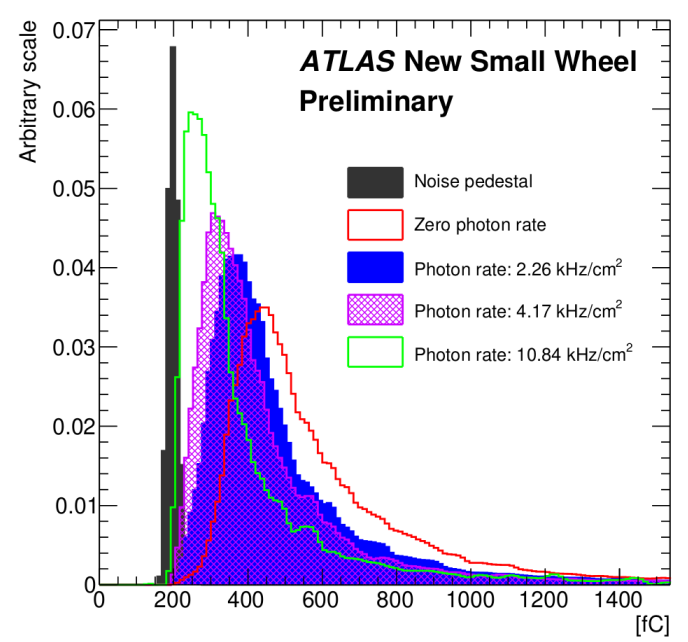

Figure 5: sTGC pad charge distribution as a function of the photon rate measured using a muon beam in the presence of a (variable) high rate photon background in GIF++ at CERN [6]. All muon histograms are normalized to unit area and the noise pedestal distribution is shown for comparison with an arbitrary scale.

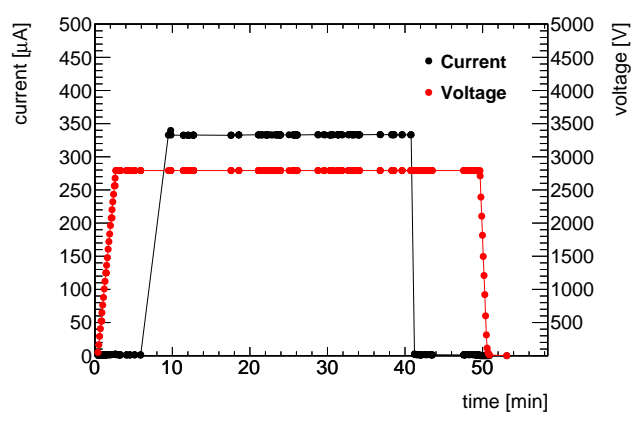

Figure 6: Irradiation test of a quadruplet (QS2) module with photons from a high intensity ${ }^{137} \mathrm{Cs}$ source during $30 \mathrm{~min}(\mathrm{t}=\sim 10-40)$. A constant current is expected during irradiation. Once the source is turned off, the current drops to 0 showing no charge build-up.

\section{3. sTGC integration}

Detector production and testing: sTGC quadruplets are produced in Canada, Chile, China, Israel, Russia and then shipped to CERN for assembly and testing. Upon reception at CERN, sTGC quadruplets undergo a series of quality assurance and quality control (QA/QC) tests to identify potential problems with the modules including: gas leak test, low-voltage test to identify broken wires, electrode connectivity test, high voltage (HV) behaviour test under high photon rate at GIF++ (Figure 6). Once assembled, sTGC wedges undergo a long-term HV stability test and the strip misalignment is measured with X-rays.

NSW commissioning status: The ATLAS New Small Wheels are being constructed in a surface building at CERN. Two sectors have been mounted on the first NSW which is scheduled to be installed in ATLAS during the current long shutdown (LS2). The sTGC production is almost complete and 23 of the 32 wedges required for the first NSW have been assembled.

\section{Conclusion}

The NSW will replace the current ATLAS inner muon end-caps in order to improve the online muon identification capabilities of the detector during operation at the HL-LHC. The sTGC wedges, making part of the NSW, are being assembled at CERN and their spatial resolution has been shown to satisfy the performance requirement of a $100 \mu \mathrm{m}$ spatial resolution. 


\section{References}

[1] ATLAS Collaboration, The ATLAS experiment at the CERN Large Hadron Collider, JINST 3 (2008) S08003.

[2] ATLAS Collaboration, New Small Wheel Technical Design Report, CERN-LHCC-2013-006, ATLAS-TDR-020, https://cds.cern.ch/record/1552862.

[3] Y. Giomataris et al., MICROMEGAS: A High granularity position sensitive gaseous detector for high particle flux environments, Nucl. Instrum. Meth. A 376 (1996) 29.

[4] S. Majewski, et al., A thin multiwire chamber operating in the high multiplication mode, Nucl. Instrum. Meth. 217 (1983) 265.

[5] B. Lefebvre, Characterization studies of small-strip Thin Gap Chambers for the ATLAS Upgrade, CERN-THESIS-2018-111, 2018, https://cds.cern.ch/record/2633639.

[6] ATLAS Collaboration, New Small Wheel Public Results, https://twiki.cern.ch/twiki/bin/view/AtlasPublic/NSWPublic Results\#Public_Plots_AN3 Accessed: 2020-05-25.

[7] D. Pfeiffer et al., The radiation field in the Gamma Irradiation Facility GIF++ at CERN, Nucl. Instrum. Meth. A 866 (2017) 91.

[8] A. Abusleme et al., Performance of a Full-Size Small-Strip Thin Gap Chamber Prototype for the ATLAS New Small Wheel Muon Upgrade, Nucl. Instrum. Meth. A 817 (2016) 85. 